\title{
Tracking Position Control of Electrohydraulic System with Minimum Number of Sensors
}

\author{
Lilia SIDHOM ${ }^{1}$, Mohamed SMAOUI ${ }^{2}$, Xavier BRUN ${ }^{2}$ \\ ${ }^{1}$ Research Laboratory LA.R.A, ENIT, \\ BP 37, Le Belvédère, Tunis, 1002 Tunisia, \\ lilia.sidhom@gmail.com \\ ${ }^{2}$ AMPERE Laboratory UMR 5005, INSA of Lyon, \\ 20 Avenue Albert Einstein, Villeurbanne, Lyon, F 69621, France, \\ xavier.brun@insa-lyon.fr, Mohamed.smaoui@insa-lyon.fr
}

\begin{abstract}
In general, feedback controllers require measurements of velocity and acceleration for feedback. To avoid the complexity of overall system with minimizing the sensor number implements on a test bench, a new estimation algorithm of the successive derivatives of measured signal is proposed. The differentiator design is a very difficult task because the important problem in real time differentiation signal is to combine differentiation exactness with robustness in respect of measurements errors and input noises. For this target, a higher order sliding modes differentiator with dynamic gains is proposed. The experimental validation highlights the performances of the differentiator/controller design of a high dynamic electrohydraulic servo-system. For this validation, two controllers are implemented on the test bench for position tracking objective. Moreover, two kinds of differentiators are also tested: the proposed one and some classic algorithm.
\end{abstract}

Keywords: Differentiator design, Dynamic gains, High order sliding mode, Experimental results, Electrohydraulic servo-actuator.

\section{Introduction}

Despite a large use of hydraulic system in industry applications, their control problem still remains an interesting challenge. Indeed, the dynamic behaviour of electrohydraulic systems is highly nonlinear which makes targets difficult to achieve. In practice, the most common type of control used for hydraulic servo-systems is a linear controller which is designed basing on the linear approximated model at an equilibrium point, [20], [17]. However in such work, some important dynamic information may be lost. Therefore, it is judiciously to choose a nonlinear control method to benefit greatly from the application of advanced control techniques, [7]. In recent years, a various studies of modern control technique have been applied on electrohydraulic actuators. Some number of them has rested on feedback linearization techniques, [23]. Other nonlinear approaches based on neural or fuzzy algorithms are also applied, [30], an adaptive controller is considered too for an electrohydraulic system [9], [15], [31]. An alternative approach have been investigated which is based on classic sliding mode which is added to the adaptive technique, [2]. For strict-feedback model system, a nonlinear controller can be designed with a Backstepping technique, [8]. This approach has been used in this paper in the context of electrohydraulic application.

All of the previous mentioned feedback controllers require generally measurements of velocity and acceleration for feedback. However, accelerometers are seldom used in practical drive systems. Indeed, the use of accelerometers adds cost, energy consumption, increases the complexity of the overall system, and reduces its reliability. Then all controllers are highly sensitive to noisy, inaccurate or delayed velocity and acceleration estimates. Nowadays, the problem of differentiation signal given in real time is an old and wellknown problem, but it still remains an important challenge. Sometimes the synthesis of a differentiator requires a good knowledge of the model system. In this case the differentiator synthesis is reduced to an observation and a filtration problem. Many structures for state variables estimation are based on nonlinear observer theory, such as high gain observer [10], sliding mode observer [24] and Backstepping observer [14]. However, the lack information or insufficient knowledge on the dynamics of the system makes the implementation of a nonlinear state observer difficult. Another attractive method for estimation of the state variables, particularly for mechanical systems, is the numerical algorithms. In [12] the properties and the 
limitations of two different structures for linear differentiation have been discussed. For example, a predictive algorithm applied to angular acceleration measurements is presented in [28].

In other cases, the construction of a differentiator is inevitable. Indeed, differentiators are very useful tools to determine and estimate signals without basing on the dynamics system. The design of differentiator unit is a traditional aim for signal processing theory. For instance, using differentiator unit, the velocity and acceleration can be computed only from the position measurements. But, the design of an ideal differentiator is a hard and challenging task. For construction a differentiator, some features of the signal and the noise must be considered. However, in some cases the structure of the signal may be unknown except some differential inequalities, differentiators that are based on algebraic parametric estimation techniques can be well employed, [21]. Although the algebraic algorithms allow a good capability to attenuate efficiently the noise, they are sensitive to the truncation order also to the size of the sliding window estimation and essentially to the setting of its parameters.

Alternative methods based on the higher order sliding mode technique can be used [27]. In [18], a robust first order differentiator via second order sliding modes is proposed. Other works, [19]; employs an arbitrary-order robust exact differentiator with finite-time convergence. The main advantage of such differentiators is the easiness of its implementation in real time.

Even though large applications of these kinds of differentiators have been performed, its major drawback concerns the tuning of its gains in real time. This adjustment requires the exactly knowledge beforehand of the Lipschitz constant of the derivative signal. Moreover, it is so difficult to obtain in advance the value of this constant in real time since we do not necessarily know the signal to estimate. In the prior researches, different new schemes of sliding modes differentiators have been proposed to improve the performance of basic schemes. Some works that can be cited are: [1], [6], [31]. In these last, a new forms of the firstorder differentiator are proposed.
In the current paper, we are going to develop the results from [19] in order to propose novel scheme of second-order differentiator which is based on third-order sliding mode. A dynamic are added to the differentiator gains to adjust them in real time and to avoid the condition of knowledge of the Lipschitz constant of Levant's differentiator. Moreover, in all the previous works, generally, all contributions that have been made relate to the first order estimator which is based on the second order sliding mode and as for the made real-time applications. Our contributions consist to: 1) Present a new extended form of the $2^{\text {nd }}$-order sliding modes differentiator to estimate simultaneous the velocity and the acceleration of the electro-hydraulic system, not the $1^{\text {st }}$ order what is usually done in the literature. 2) Synthesis and implement a Backstepping controller on high dynamic electro-hydraulic system for a tracking position trajectory. 3) Discuses experimental results with different desired trajectories of position to show the performance of the proposed algorithm with compare it to some classical numerical differentiation algorithm.

The paper is organized as follows. The first section outlines the $2^{\text {nd }}$-order Adaptive Differentiator (ARD) is developed. Section2 describes the model of the electrohydraulic actuator. Section 3 presents the controller design via a Backstepping technique for position tracking trajectory. Section 4 is devoted to the experimental results.

\section{Differentiator Construction via Higher Order Sliding Modes}

The differentiation algorithm must ensure a trade-off between the noise level of the output signal and its phase shift relative to the exact derivative signal. Some of those algorithms are based on the technique of sliding modes. This technique is mostly used to elaborate the control laws [5], [16], [19]. The main features of the sliding modes control are its robustness to variation in system parameters, external disturbances and modelling errors, [27]. For all the above reasons, this technique shows good results in the synthesis and the implementation of robust differentiators, [19], such as the Super Twisting algorithm, [18]. In practice, the major problem associated with this algorithm is the tuning up of the convergence gains of the 
differentiator. It is not always easy to determine these gains for a given bandwidth of the input signal. A simple modification of the spectral content of the input signal or its amplitude can cause a significant error in the estimation of the derivative. Indeed, the parameters of the algorithm strongly depend on the Lipschitz constant of the $\mathrm{n}^{\text {th }}$ derivative of the input signal and, generally this constant is not accurately known beforehand. This problem can be resolved with adding an adaptive law to the gains of the classical Super Twisting algorithm.

In this paper, we have chosen to use a second order differentiator, rather than using several cascade first order differentiators for a better estimation. The differentiator is based on a recursive diagram of arbitrary-order robust differentiators with finite time convergence proposed in [19]. However, the number of gains related to this method is more important than a Super Twisting. So the main problem of this recursive algorithm is actually related to the choice of the differentiator parameters. We must choose judiciously the gains of this algorithm to derive an input for which the spectrum has rich frequencies. The fact that these parameters depend on the input signal is an effective limit of the method performances.

The accuracy of this classic robust algorithm depends on the choice of three parameters. Moreover, the choice of these parameters is difficult. Furthermore their choices are not to be too large in order not to be differentiating the noise. So the most suitable parameters can be defined by using the adaptive mechanism, in order to regulate these gains of such algorithm.

Consider an input signal of differentiator $f(t)$ as a function defined on $[0, \infty[$ measurable in Lebesgue's sense which can be considered as the sum of two following terms

$$
f(t)=f_{0}(t)+\xi(t)
$$

$f_{0}(t)$ is an unknown clear-off-noise signal with the $(\mathrm{n}+1)^{\text {th }}$ derivative having a known Lipschitz constant $C>0 . \zeta(t)$ is a bounded Lebesgue-measurable noise with unknown features and it is defined by: $|\xi(t)|<\varepsilon$, with $\varepsilon$ is sufficiently small so $\left|f(t)-f_{0}(t)\right| \leq \varepsilon$.

For the 2nd-order differentiator, three gains $\left(\hat{\lambda}_{1}, \hat{\lambda}_{2}, \hat{\lambda_{3}}\right)$ must be adjust in real time. Let us define now the schema of the $2^{\text {nd }}$ order differentiator by system of equations (2).

$$
\left\{\begin{array}{l}
\dot{z}_{0}=v_{0} \\
v_{0}=-\hat{\lambda}_{0}\left|s_{0}\right|^{\frac{2}{3}} \operatorname{sign}\left(s_{0}\right)+z_{1}-K_{0} s_{0} \\
\dot{z}_{1}=v_{1} \\
v_{1}=-\hat{\lambda}_{1}\left|s_{1}\right|^{\frac{1}{2}} \operatorname{sign}\left(s_{1}\right)- \\
\quad-\hat{\lambda}_{2} \int_{0}^{t} \operatorname{sign}\left(s_{1}\right) d t-K_{1} s_{1}
\end{array}\right.
$$

$\hat{\lambda}_{1}, \hat{\lambda}_{2}, \hat{\lambda}_{3}$ are dynamic gains, $K_{0}$ and $K_{1}$ convergence gains and $s_{0}, s_{1}$ the sliding surfaces defined as:

$$
\left\{\begin{array}{l}
s_{0}=z_{0}-f \\
s_{1}=z_{1}-v_{0}
\end{array}\right.
$$

Function $\operatorname{sign}($.$) is defined by:$

$$
\operatorname{sign}(.)=\left\{\begin{aligned}
1 & \text { for }(.) \geq 0 \\
-1 & \text { otherwise }
\end{aligned}\right.
$$

The dynamic gains $\hat{\lambda}_{i}, i \in\{0,1,2\}$ are defined by:

$$
\left\{\begin{array}{c}
\dot{\hat{\lambda_{0}}}=\left[\left|s_{0}\right|^{\frac{2}{3}} \operatorname{sign}\left(s_{0}\right)\right] s_{0}, \\
\hat{\lambda_{0}}(0) \geq 0 ; \dot{\hat{\lambda}_{0}}>0 \forall t>0 \\
\dot{\hat{\lambda}_{1}}=\left[\left|s_{1}\right|^{\frac{1}{2}} \operatorname{sign}\left(s_{1}\right)\right] s_{1}, \\
\hat{\lambda}_{1}(0) \geq 0 ; \dot{\hat{\lambda}_{1}}>0 \forall t>0 \\
\hat{\lambda_{2}}=s_{1} \int_{0}^{t} \operatorname{sign}\left(s_{1}\right) d t
\end{array}\right.
$$

Theorem. For $K_{0}, K_{1}>0$ and with the dynamic gains $\hat{\lambda}_{i}, i \in\{0,1,2\}$ defined by (5), the system trajectories (2) converge locally and asymptotically towards the equilibrium point $s_{0}=s_{1}=0$ under the assumption that as there are a positive constants $\lambda_{0}^{*}, \lambda_{1}^{*}$ and $\lambda_{@}^{*}$, a priori unknown, defined by:

$$
\left\{\begin{array}{l}
\ddot{f}=-\lambda_{0}^{*}\left|s_{0}\right|^{\frac{2}{3}} \operatorname{sign}\left(s_{0}\right)+z_{1} \\
\ddot{f}=-\lambda_{1}^{*}\left|s_{1}\right|^{\frac{1}{2}} \operatorname{sign}\left(s_{1}\right)-\lambda_{2}^{*} \int_{0}^{t} \operatorname{sign}\left(s_{1}\right) d t
\end{array}\right.
$$

See the proof and the details of the previous theorem are given in [25].

\section{Electrohydraulic Model System}

The considered system (see Figure 1) is a symmetric double acting electro-hydraulic servodrive using a double-rod cylinder controlled by 
two five-way servo-valves. Table 1 shows the specifications of the hydraulic actuator.

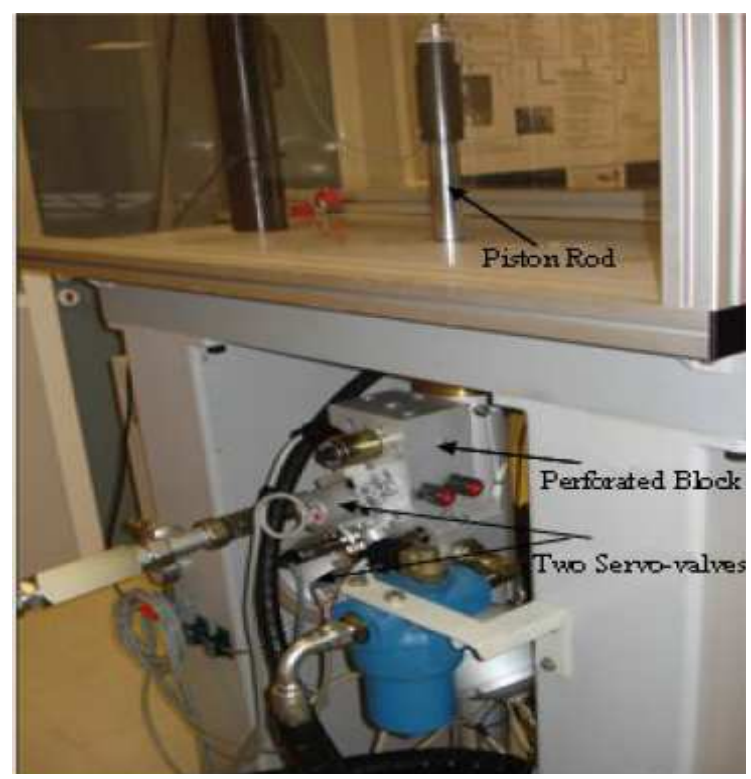

Figure 1. Electrohydraulic test bench.

Table 1. Specification of the hydraulic actuator.

\begin{tabular}{|l|l|}
\hline Diameter of piston & $50 \mathrm{~mm}$ \\
\hline Diameter of rod & $30 \mathrm{~mm}$ \\
\hline Total moving load & $5.9 \mathrm{~kg}$ \\
\hline
\end{tabular}

The used power modulators are an electrohydraulic servomechanism that constitutes the main interface between the electrical control signal and the fluid power actuator, which integrated a two stage. These modulators developed by MOOG, can provide a quite large bandwidth, thus a short response time, and a good precision. As indicated in the MOOG technical bulletin, the bandwidth reaches to $1 \mathrm{kHz}$ for $5 \%$ of spool displacement with a rated flow up to $19 \mathrm{l} / \mathrm{min}$ at 70 bars pressure drop for full opening. The perforated block was conducted specifically for this test-bench. It allows implement the two servo-valves and other components in order to ensure different operating mode of system. Thus the actuator chambers can be fed either by a $5 / 2$ single servo-valve or by two servo-valves in parallel to increase throughput, or use its in two threeway mode to supply only one single actuator chamber by one servo-valve. To enable this modularity, the perforated block is equipped with two electro-distributors and two regulated flow orifice. Finally, to filter the pressure variation upriver of servo-valves, two spherical accumulators are also mounted on this block. The presence of this perforated block enhances versatility to the test-bench. However, this interface introduces parasite phenomenon (drop pressure, capacitive and inertial effects) that could present a significant influence on the overall behaviour of the system, in some circumstances of use.

In this paper, a single mode is considered which consist to control the actuator by two $5 / 2$ servo-valves in parallel. The proposed model used for synthesizing the control law rest on some assumptions. The first one consists to take into account only the resistive effect produced by the interface bloc. This hypothesis will be justified later by the trajectory choice.

According to second law of Newton, the dynamic equation of the inertia load is given by:

$$
M a=S \Delta p-M g-b v+h(v(t))
$$

where $M$ is the mass of the moving part, $\Delta p=p_{1}-p_{2}$ is the drop across load piston $S$ is the effective area of the actuator chambers, $b$ represents the coefficient of the viscous friction force, $v, a$ are respectively the velocity and the acceleration of the load. Dry friction force depend explicitly by velocity which is represented by the function $h(v(t))$. In our work, nonlinear model will be adopted basing on the well-known Tustin friction model [26]. The proposed model is given by

$$
\begin{aligned}
& h(v(t))= \\
& \quad=\left[F_{\text {sdyn }}+\left(F_{\text {sdyn }}-F_{C}\right) e^{(-C \mid v)}\right] \tanh (v(t))
\end{aligned}
$$

The flow evolution law in a chamber with variable volume which depends on the piston position $y$ is obtained assuming the following assumptions:

i) Both the temperature and the pressures of the oil are supposed to be homogeneous in each chamber.

ii) Neglecting the variation density to the average density.

iii) Assuming that the temperature variation is negligible with respect to average and equal to the supply temperature.

In our work, the two servo-valves are considered as identical and a symmetrical one, which are controlled by the same input signal $u$. These power modulators present a large bandwidth compared to the dynamics of the actuator. Neglecting the laminar flow of fluid, the flow laws defined can be written to give an expression of the laws used for actuator input $\left(Q_{1}, Q_{2}\right)[20]$ : 


$$
\left\{\begin{array}{l}
Q_{1}=R \cdot \chi_{1}\left(p_{1}, p_{P}, p_{T}, \operatorname{sign}(u)\right) u \\
Q_{2}=R \cdot \chi_{2}\left(p_{2}, p_{P}, p_{T}, \operatorname{sign}(u)\right) u
\end{array}\right.
$$

Where $R$ is a parameter that implicitly depends on the feature of the system and the pressure drop induced by the perforated block. This variable $R$ is considered a constant one and is identified experimentally for a turbulent flow. With $p_{p}, p_{t}$ are respectively the supply pressure and the exhaust pressure of the fluid.

Where,

$$
\left\{\begin{aligned}
\chi_{1}(.) & =\gamma(u) \sqrt{p_{P}-p_{1} \mid} \operatorname{sign}\left(p_{P}-p_{1}\right)+ \\
& +\gamma(-u) \sqrt{p_{1}-p_{T} \mid} \operatorname{sign}\left(p_{1}-p_{T}\right) \\
\chi_{2}(.) & =\gamma(u) \sqrt{p_{2}-p_{T} \mid} \operatorname{sign}\left(p_{2}-p_{T}\right)+ \\
& +\gamma(-u) \sqrt{\left|p_{P}-p_{2}\right|} \operatorname{sign}\left(p_{P}-p_{2}\right)
\end{aligned}\right.
$$

The function $\gamma(u)$ is given by $\gamma(u)=\frac{1+\operatorname{sign}(u)}{2}$, where “ $\operatorname{sign}($.$) " is$ defined as in (4).

Under normal practical operating conditions, the physical domain of system can be described by:

$$
\begin{aligned}
D_{0}=\{(y, v, \Delta P) & \in I R^{3} /|y| \leq \frac{l}{2}, \\
& \left.p_{1}, p_{2} \in \Omega_{p} \equiv\right] p_{T}, p_{P}[\}
\end{aligned}
$$

Using (16), the terms $\operatorname{sign}\left(p_{P}-p_{j}\right)$ and $\operatorname{sign}\left(p_{j}-p_{T}\right)$ with $j=\{1,2\}$, introduced in (15) can be eliminated. Define the state variable as: $X=[y, v, a]^{T}$. Thus, the model system can be defined by a nonlinear affine form with a single input as follows:

$\dot{X}=f(X)+g(X) u$

with $X, f(X), g(X) \in I R^{3}$ and $u \in I R$

$$
\begin{aligned}
& f(X)= \\
& =\left(\begin{array}{c}
v \\
a \\
-\frac{b}{M} a-\frac{\dot{h}(v(t))}{M}-\frac{S^{2} \beta}{M}\left(\frac{1}{V_{1}(y)}+\frac{1}{V_{2}(y)}\right) v
\end{array}\right)
\end{aligned}
$$

and

$$
g(X)=\left(\begin{array}{c}
0 \\
0 \\
\frac{S \beta}{M}\left(\frac{R \chi_{1}(.)}{V_{1}(y)}+\frac{R \chi_{2}(.)}{V_{2}(y)}\right)
\end{array}\right)
$$

$V_{1}(y)$ and $V_{2}(y)$ are the total volumes of the cylinder, defined respectively by:

$$
\left\{\begin{array}{l}
V_{1}(y)=V_{0}+S y \\
V_{2}(y)=V_{0}-S y
\end{array}\right.
$$

where: $V_{0}=V_{D}+\frac{S l}{2}$ is the piping volume of the chambers for the zero position, $V_{D}$ is a dead volume present on each extremities of the cylinder, $y$ is the displacement of the load and $l$ is the cylinder stroke.

\section{Controller Design}

Note that $y_{d}, v_{d}, a_{d}$ are respectively the desired trajectories for the position velocity and acceleration. Our objective is to find the control law $u$ in order to track a desired position $y_{d}$.

With different steps of design a Backstepping controller, it is so simple to obtain the general form of $u$ as following:

$$
\begin{gathered}
u=\frac{1}{\frac{S \beta}{M}\left[\frac{R \chi_{1}(.)}{V_{1}(y)}+\frac{R \chi_{2}(.)}{V_{2}(y)}\right]}\left[-f_{3}(.)+\dot{a}_{d}-\right. \\
\left.-k_{1} e_{1}-k_{2} e_{2}-k_{3} e_{3}\right]
\end{gathered}
$$

Where

$$
\left\{\begin{array}{l}
k_{1}=2-c_{1}^{2}-c_{2}^{2}-c_{1} c_{2}, \\
k_{2}=c_{1}^{3}-2 c_{1}-c_{2}, \\
k_{3}=c_{1}+c_{2}+c_{3} .
\end{array}\right.
$$

With $c_{1}, c_{2}, c_{3}>0$.

From the different variables errors defined during the construction of the control law, this error system is then defined:

$$
\left\{\begin{array}{l}
\dot{e}_{1}=-c_{1} e_{1}+e_{2} \\
\dot{e}_{2}=e_{3}-c_{2} e_{2}-e_{1} \\
\dot{e}_{3}=\dot{a}-\dot{\alpha}_{i}\left(e_{1}, e_{2}, y_{d}, v_{d}, a_{d}\right)= \\
\quad=f_{3}(.)+g_{3}(.) u-\dot{\alpha}_{i}(.)
\end{array}\right.
$$

With

$$
\begin{aligned}
f_{3}(X) & =-\frac{b}{M} a-\frac{\dot{h}(v(t))}{M}- \\
& -\frac{S^{2} \beta}{M}\left(\frac{1}{V_{1}(y)}+\frac{1}{V_{2}(y)}\right) v \\
g_{3}(X) & =\frac{S \beta}{M}\left(\frac{R \chi_{1}(.)}{V_{1}(y)}+\frac{R \chi_{2}(.)}{V_{2}(y)}\right)
\end{aligned}
$$

The Lyapunov function of the overall system is given by : 


$$
V\left(e_{1}, e_{2}, e_{3}\right)=\frac{1}{2} e_{1}^{2}+\frac{1}{2} e_{2}^{2}+\frac{1}{2} e_{3}^{2}
$$

It is clear that the singularity of the equation (16) happens when $\chi_{1}()=.\chi_{2}()=$.0 , which can be occur when the pressure in the two chambers are equal respectively to the supply and the exhaust pressure.

\section{Experimental Result}

Experiment results are provided here to demonstrate the effectiveness of the Differentiator/controller Design on the electrohydraulic servo-system.

Results of the Backstepping control law are also compared with those given by a linear state-feedback controller. Both control laws are implemented using a Dspace 1104 controller board with the dedicated digital signal processor. The sampling frequency of the control loop is equal to $1 \mathrm{kHz}$. The sensed signals, all analogs were passed through the signal conditioning unit in order to filtrate and amplify these analog outputs before being supplied to the Analog/Digital Converters. Five sensors are implemented on the test bench: position sensor and four pressures sensors. Two of them are related to measuring the pressure level of cylinder chambers; there the static error with combining nonlinearity and hysteresis is equal to $0.1 \%$. The two other pressure sensors are used respectively for measuring the supply and the exhaust pressure. The precision for the supply pressure sensor is equal to $0.15 \%$ and it is equal to $0.25 \%$ for the exhaust pressure sensor. The position is measured by an LVDT measurement with a maximal displacement is equal to $330 \mathrm{~mm}$.

There are two methods to obtain velocity and an acceleration feedback, namely using sensors to measure them or using position information to generate the both. In the last case, the differentiation method represents the key step that helps a best estimation of the derivative of measured state variable. In order to use a minimum number of sensors on the test bench, a proposed ARD is used to recover the velocity and the acceleration signal.

A linear state-feedback control with fixed-gains is also implemented on the servo-system. This controller is based on the local linearization of the nonlinear dynamics' system around a nominal operating point. So the state-feedback linear control can be synthesized with position, velocity and acceleration, which are defined as state variables. Then, this linear control law can be given by the following equation:

$u_{1}=u_{e}+K_{y}\left(y-y_{d}\right)-K_{v} v-K_{a} a$

with $K_{y}, K_{v}$ and $K_{a}$ are respectively a feedbackgains of position, velocity and acceleration. The coefficients of the feedback state are setting in order to obtain the dynamics of the closed loop system as a canonical Brunovsky form [4].

The aim of the synthesized control law is to keep a good accuracy in terms of the position tracking trajectory. The relative degree of the position is equal to three. This means that the electrohydraulic system can only track position trajectory at least three times differentiable. The desired trajectory has been carefully chosen in order to respect the differentiability required. In addition, this trajectory must be chosen to satisfy the assumptions already considered when performing model system. Two types of trajectories are used. First, a sinusoidal trajectory is defined with respectively a frequency and a maximal magnitude of displacement equals to $0.5 \mathrm{~Hz}$ and $330 \mathrm{~mm}$ (see Figure 2). The dynamics of the actuator around $30 \mathrm{~mm}$ as position is equal to $285 \mathrm{~Hz}$. Therefore, the dynamics of the servo-valves may well be neglected compared to the actuator.

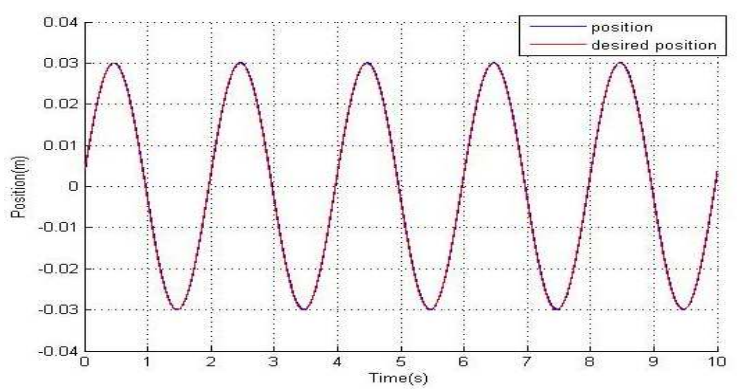

Figure 2. Position and desired position with $\mathrm{u}_{1}$

During the modeling phase, both capacitive and inertial effects due to the presence of the perforated block are not considered. In effect, for trajectories at low frequencies, the inertial effect is neglected. Moreover, the variation of the chambers volumes generated by the imposed trajectory is considered dominant over the volume of fluid in the perforated block.

\subsection{Results with the linear controller}

In the first way, a closed loop controller which designed by equation (22) is performed while the system is tracking a sinusoidal signal. 
Figures 2, 3 and 7 are show the position and the desired position, the position error and the control input which obtained by the linear controller computed with using the ARD. In order to prove the effectiveness of the new form of the robust differentiator, a comparative study with some classic algorithm (23) is carried out which is given by following expression:

$$
\operatorname{output}(k)=\frac{\operatorname{input}(k)-\operatorname{input}(k-2)}{2 T_{e}}
$$

with $T_{e}$ is a sampling period.

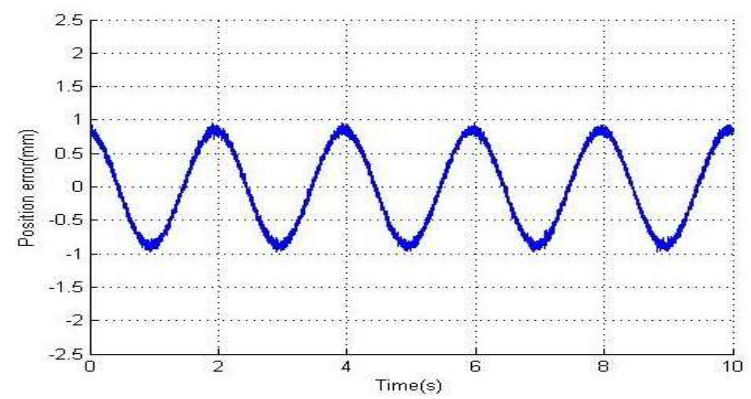

Figure 3. Position error for $u_{1}$ : with ARD

Figures 4, 5 and 6 illustrate the velocity and the acceleration which are computed by the both methods, the ARD and the classic algorithm. In effect, from the measured position, the velocity is building once by the classic scheme and once by the ARD (sees Figure 4). With using the cascade form of classic algorithm, the acceleration is also estimated (see Figure 4). From Figure 6, the second output of the unit proposed differentiator is given. From these figures, it is noteworthy that the estimation of the outputs obtained with the ARD is less noisy than the classic algorithm, especially for the estimation of the acceleration (see Figure 5). Therefore, the adaptive differentiator is insensitive to high frequency components of the position signal and consists to obtain accurate outputs. For each method of differentiation, the calculated outputs are injected into the control law (see Figure 7). Clearly the control input computed with using the classic scheme is more affected by the chattering phenomenon, what is due to the velocity and mostly the acceleration signal. From this figure and with using the classic algorithm, the control input does not present a high level of chattering in comparison to the one found on the velocity and acceleration estimated signal (see Figures 5 and 4). This is explained by the low velocity and acceleration gains imposed at the control law. In fact, the large values of gains lead to a high position

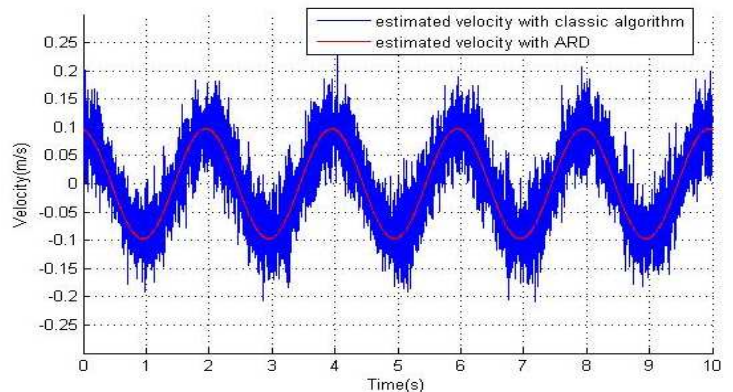

Figure 4. Velocity estimated by both ARD and the classic algorithm

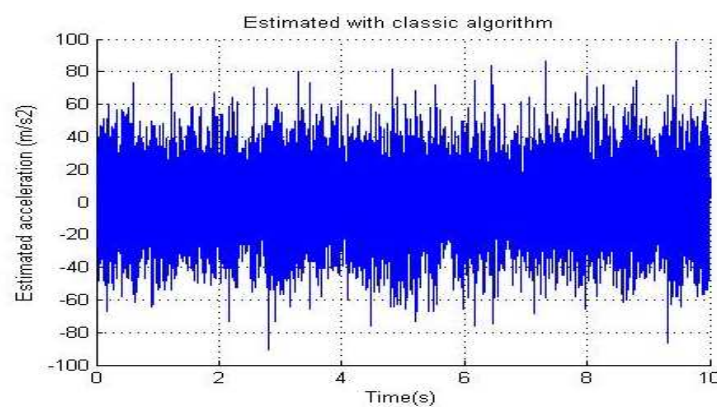

Figure 5. Estimated acceleration with classic algorithm

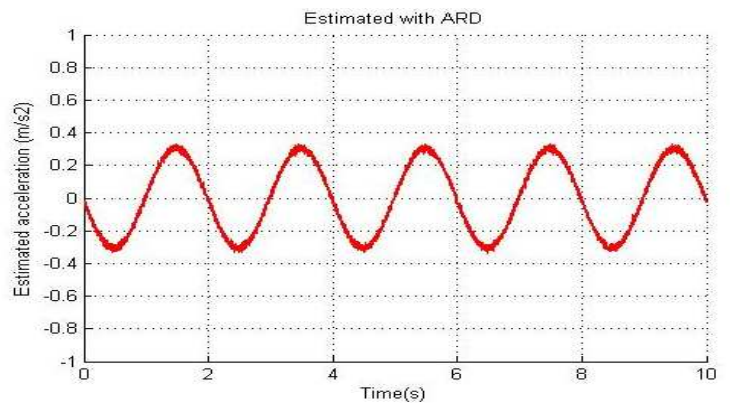

Figure 6. Estimated acceleration with ARD

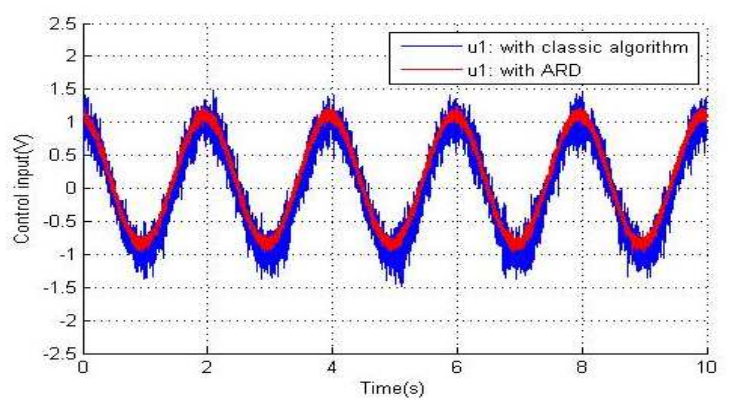

Figure 7. Control input $u_{1}$

error. For this reason, the Figure 7 does not show very well the difference between the two differentiation methods. Figure 6 illustrates the evolution of the error position with using the ARD, for the state-feedback controller. Then the resulted average position error is about $0.85 \mathrm{~mm}$.

\subsection{Results with the nonlinear controller}

Now, the results of Backstepping controller defined by (16) are presented with using only the ARD for recover the velocity and the 
acceleration signal. In this case, using the classical scheme is impossible since the velocity and the acceleration gains are respectively about 20 and 10 which amplifies enormously chattering effect. This control law depends on three parameters $c_{1}, c_{2}$ and $c_{3}$, which must be tuning to ensure an acceptable tracking trajectory. As is shown in Figure 8, the average position error is about $0.39 \mathrm{~mm}$. This value is twice times less than the error value given by linear control law. This can explain the importance of the consideration of the nonlinearities system and especially the resistive effect that is generated by the perforated block. Moreover, a good tracking trajectory is observed in Figures 9 and 10. Figure 11 shows the evolution of the control law $u$. In the second way, another kind of trajectory is used. This trajectory is defined by a fifth-order polynomial function. The amplitude of displacement is equal to $50 \mathrm{~mm}$ which represents $0.15 \%$ of the total stroke. The maximum desired velocity is equal to 0.0938 $\mathrm{m} / \mathrm{s}$. Figures 12 and 14 present the evolution position error given by both control laws. In static stage, the mean position error for the state-feedback controller is about $80 \mu \mathrm{m}$. For the controller based on a Backstepping technique, this value is equals to $40 \mu \mathrm{m}$. This low value of the static error is explained by the low value of dry friction. Since at very low velocities, a partial lubrication regime is reached. So the dry static friction is negligible in comparison to other effects as the viscous and the Coulomb effect. In dynamic stage, the maximum position tracking error is about 0.94 $\mathrm{mm}$, for the linear controller compared to 0.38 $\mathrm{mm}$ given by the nonlinear one. This value is nearly twice times less than the value founded by the state-feedback strategy law.

With using the ARD, the control laws evolutions are without high-frequency excitation (see Figures 13 and 15), which is a good property from an energetically efficiency point of view. Figures 16 and 17 represent respectively velocity and desired velocity, acceleration and desired acceleration. Indeed, these successive derivatives of position output are filtered and introduce practically no phase shift. From the experimental results, the best performances are obtained using Backstepping controller in terms of position tracking.

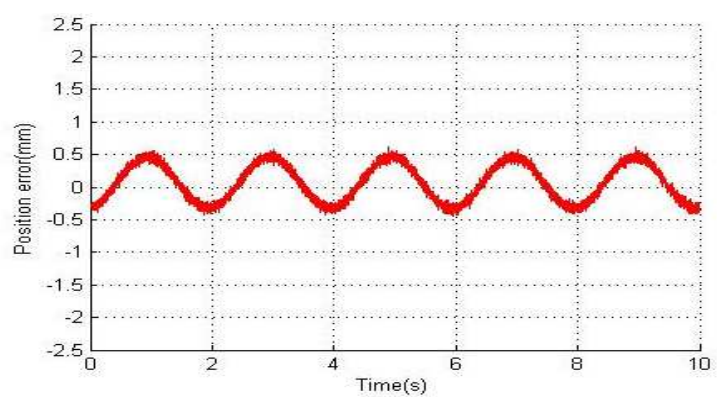

Figure 8. Position error with the Backstepping controller.

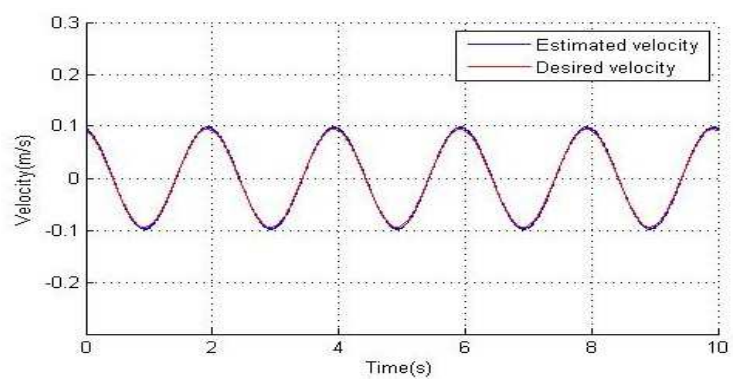

Figure 9. Desired velocity and estimated velocity with ARD

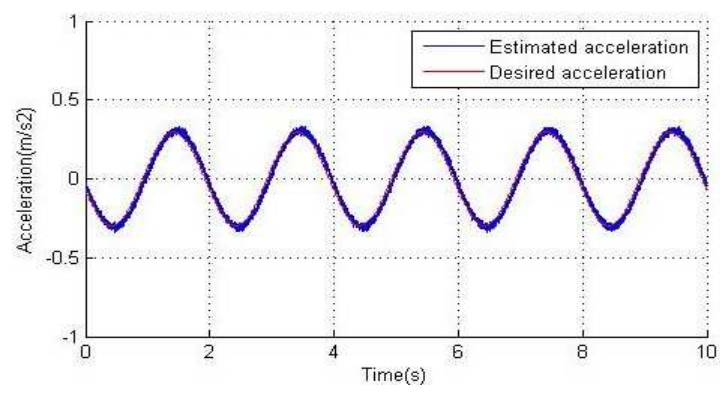

Figure 10. Desired acceleration and estimated acceleration with ARD.

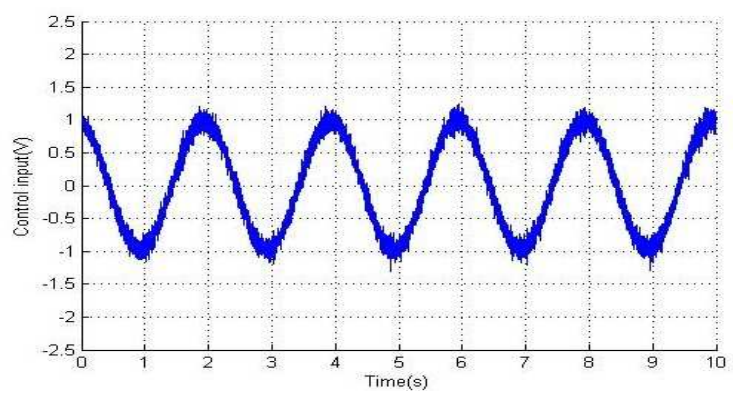

Figure 11: Control input $u$.

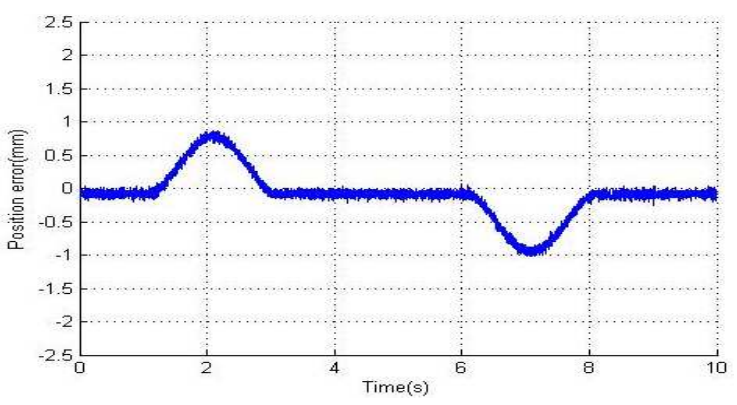

Figure 12. Position error with the feedback linear controller. 


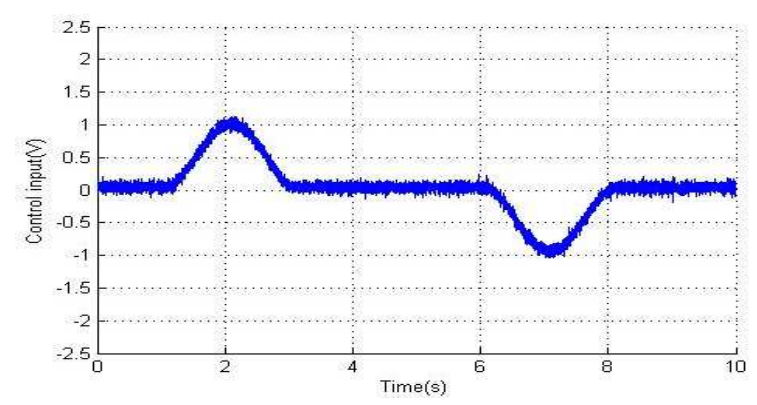

Figure 13. Control input $u_{1}$.

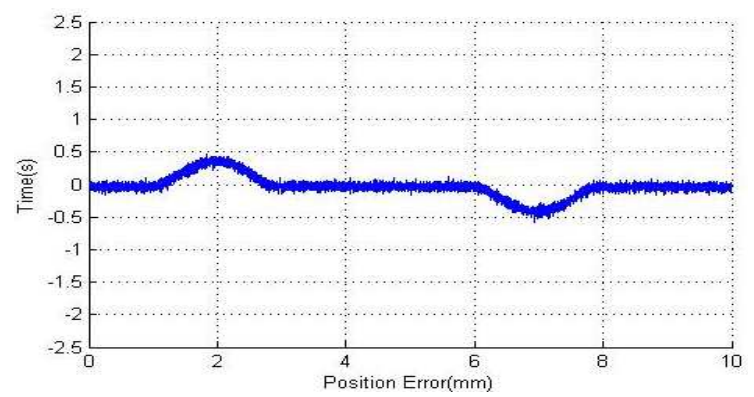

Figure 14. Position error with the Backstepping controller.

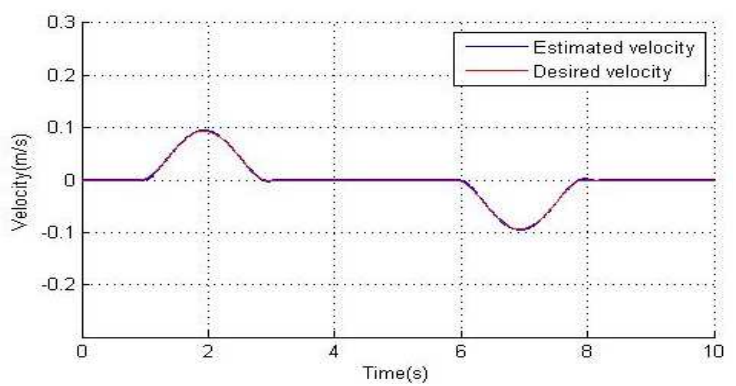

Figure 15. Desired velocity and estimated velocity with ARD.

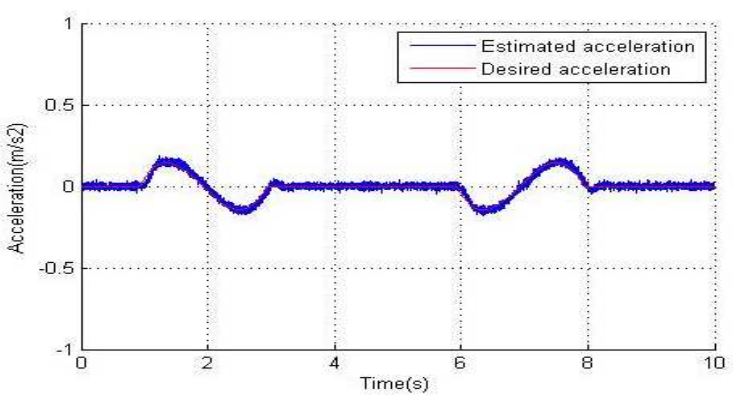

Figure 16. Desired acceleration and estimated acceleration with ARD.

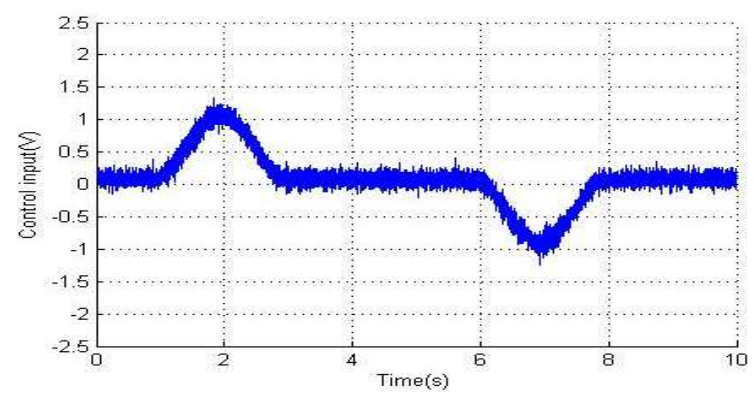

Figure 17: Control input $u$.

\section{Conclusions}

In this paper, a unit adaptive robust differentiator has been proposed in order to compute the successive derivatives of the position. The direct validation highlights the performances of the proposed algorithm. Experimental results are carried out in order to show the important to the choice differentiator design on the control of the high dynamic electro-hydraulic system. The adaptive robust algorithm shows a satisfactory result compared to some other classic differentiator. The algorithm efficiently attenuates the noise for these two outputs.

\section{REFERENCES}

1. ANGUlO, M. T., J. A. MORENO, L. FRIDMAN, The Differentiation Error of Noisy Signals Using the Generalized Super-Twisting Differentiator, 51st IEEE Conf. on Dec. \& Ctrl., Maui, 2012.

2. ALLEYNE, A., R. LUI, Systematic control of a Class of Nonlinear Systems with Application to Electrohydraulic Cylinder Pressure Control, IEEE Trans. on Ctrl. Syst. Tech., 6(4), 2000, 623-634.

3. ARMSTRONG-HELOUVRY, B., P. DUPONT, C. CANUDAS De WIT, C. A Survey of Models, Analysis Tools and Compensation Methods for the Control of Machines with Friction, Automatica, 30 (7), 1994, 1083-1138.

4. BLACKBURN, J. F., G. REETHOF, J. L. SHEARER, Fluid Power Control, 1960. M.I.T Technology Press, and Wiley.

5. BOURI, M., D. THOMASSET, Sliding Control of an Electro-pneumatic Actuator Using an Integral Switching Surface, IEEE Trans. on Ctrl. Syst. Tech., 9(2), 2001, 368-375.

6. CHAWDA, V., O. CELIK, M. K. O'MALLEY, Application of Levant's Differentiator for Velocity Estimation and Increased Z-Width in Haptic Interfaces, IEEE World Haptics Conf., June, Istanbul, 2011.

7. EDGE, K. A. The Control of Fuid Power Systems Responding to the Challenge, J. Syst. \& Ctrl Eng., 211(12), 1997, 91-110. 
8. FREEMAN, R. A., P. KOKOTOVIC, Desgin of Softer Robust Nonlinear Control Laws, Automatica, 29, 1993, 1425-1437.

9. GUAN, C., S. PAN, S. Adaptive Sliding Mode Control of Electrohydraulic System With Nonlinear Unknown Parameters, J. Ctrl Eng. Prac., 2008, 16, 1275-1284.

10. GAUTHIER, J. P., H. HAMMOURI, S. OTHMAN, A Simple Observer for Nonlinear Systems Applications to Bioreactors, IEEE Trans. Aut. Ctrl., 37(37), 1992, 875-880.

11. IBRIR, S., New Differentiators for Control and Observation Applications, Proc. Am. Ctrl. Conf., 2001, 2522-2527.

12. IBRIR, S. Linear Time-Derivative Trackers, Automatica, 2004, 40, 397-405.

13. KANELLAKOPOULOS, I., P. KOKOTVIC, A. MORSE, Systematic Design of Adaptive Controllers for Feedback Linearizable Systems, IEEE Trans. Aut. Ctrl., 36, 1991, 1241-1253.

14. KRENER, A. J., W. KANG, Locally Convergent Nonlinear Observers, Siam J. Ctrl. Optim., 42(1), 2003, 155-177.

15. KRSTIÇACUTE, M., L. KANELLAKOPOULOS, P. KOKOTOVIÇCUTE, Non-linear and Adaptive Control Design, John Wiley \& Sons, Inc., 1995, p. 563.

16. LAGHROUCHE, S., M. SMAOUI, F. PLESTAN, X. BRUN, Higher Order Sliding Mode Control Based on Optimal Approach of an Electropneumatic Actuator, Int. J. Ctrl, 79, 2006, 119-131.

17. LAVAL, L., N. K. M'SIRDI, J.-C. CADION, $H_{\infty}$ Force Control of a Hydraulic Servo-Actuator With Environmental Uncertainties, IEEE Inter. Conf. on Rob. \& Aut., 2, 1996, 156-1571.

18. LEVANT, A., Robust Exact Differentiation via Sliding Mmode Technique, Automatica, 34, 1998, 379-384.

19. LEVANT, A., Higher Order Sliding Modes, Differentiation and Output Feedback Control, Int. J. Ctrl, 76, 2003, 924-941.

20. MERRITT, H. E., Hydraulic Control Systems. John Wiley \& Sons, 1967, p. 368.
21. MBOUP, M., C. JOIN, M. FLIESS, Numerical Differentiation with Annihilators in Noisy Environment, Num. Alg., 50(4), 2009, 439-467.

22. NIKSEFAT, N., N. SEPEHRI, Design and Experimental Evaluation of a Robust Force Controller for an Electro-hydraulic Actuator via Quantitative Feedback Theory, Ctrl. Eng. Prac., 8, 2000, 1335-1345.

23. PLUMMER, A. R., N. D. VAUGHAN, Robust Adaptive Control for Hydraulic Servosystems, J. Dyn. Syst., Meas. \& Ctrl, 1996, 118, 237-244.

24. Slotine, J. E., W. LI, Applied Nonlinear Control, Prentice Hall, 1991, p. 461.

25. SIDHOM, L., X. BRUN, M. SMAOUI, E. BIDEAUX, D. THOMASSET, Dynamic Gains Differentiator for Hydraulic System Control, J. Dyn. Syst., Meas., \& Ctrl., 137(4), 2015, 017-041.

26. TUSTIN, S., The Effects of Backlash and of Speed-Dependent Friction on the Stability of Closed-Cycle Controls System, J. Inst. El. Eng., 94, 1947, 143-151.

27. WANG, H., W. ZHANG, Y. TIAN, Q. QU, Sliding Mode Control for Diesel Engine Using Extended State Observer, Studies in Informatics and Control, 24(4), 2015, 439-448.

28. VALIVIITA, S., S. J. OVASKA, Delayless Recursive Differentiator With Efficient Noise Attenuation for Control Instrumentation, J. Sign. Proc., 34, 1998, 379-384.

29. YAO, B., F. BU, J. REEDY, G. CHIU, Adaptive Robust Motion Control of Single-Rod Hydraulic Actuators: Theory and Experiments, IEEE/ASME Trans. Mech., 5, 2000, 79-91.

30. ZAMOUM B., R., B. CHETATE, Y. ZAMOUM, Artificial Neural Network Control of the Recycle Compression System, J. St. Inf. \& Ctrl, 24(3), 2015, 301-308.

31. SKENDER, M. R., A. TLEMCANI, Implementation of a New Super Twisting Mode Algorithm Controlled by Dspace: Application to Series Multicell Converter, Studies in Informatics and Control, 25(2), 2016, 255-264. 